\title{
A stakeholder oriented approach to the optimization of transports of people with disabilities
}

\author{
Oscar Tellez ${ }^{\mathrm{a}}$, Laurent Daguet $^{\mathrm{a}}$, Fabien Lehuédéb, Thibaud Monteiro ${ }^{\mathrm{a}}$, \\ Geovanny Osorio Montoyac ${ }^{\mathrm{c}}$, Olivier Péton ${ }^{\mathrm{b}}$, Samuel Vercraene ${ }^{\mathrm{a}}$ \\ a Université de Lyon, INSA Lyon, Laboratoire DISP (EA 4570), Lyon, France; \\ b IMT Atlantique, Laboratoire des Sciences du Numérique de Nantes (LS2N, UMR CNRS 6004), Nantes, France; \\ ${ }^{\mathrm{c}}$ Ressourcial / Fondation OVE, Vaulx-en-Velin, France.
}

April 2020

\begin{abstract}
From 2010, the health transportation in France has become one of the top ten priorities of the risk management plan due to the increasing cost of these transports. For social and Medico-Social Institutions (MSI), this cost represents the second-biggest expense after that of the wages. In this context, the NOMAd project aims to improve the daily transportation service for people with disabilities between their home to MSI. To this end, we performed a field survey to identify the needs of the different stakeholders. This survey allows us to propose the transportation pooling among several MSIs on one side, and a global transport management process on the other side. This process makes possible to group and optimize routes on a given geographical area. The challenge is then to improve economic performance while maintaining social and environmental goals. A decision aiding tool for the transport optimization is proposed to tackle this problem.
\end{abstract}

Keywords: Decision support system; transport sharing; passenger transport; optimization; healthcare; field study

\section{Introduction}

In 2010, medical transportation became one of the top ten priorities of the risk management plan of the French insurance scheme due to the increasing cost of these transports ANAP (2013). In France, the sector named "transport sanitaire" includes both the medical transportation and the medico-social transportation. This sector serves both elderly and people with disabilities. The report "Establishments and services for disabled people - Offers and needs, financing modalities", published by the General Inspectorate of Social Affairs \& General Inspection of Finance estimates the transportation cost for all medico-social institutions at $10 \%$ of the total cost of medical transportation (IGF/IGAS 2012). So, in 2017, the medico-social transportation cost represents 500 million euros, at the national level, for all medico-social institutions (Gonzalez et al. 2018). There are few details on the evolution of this cost. Between 2006 and 2014, in 9 years, this cost has increased by 40\% (Lesteven et al. 2016).

For Medico-Social Institutions (MSI), transportation costs often represent the second-largest expenditure after that of the staff (ANAP 2016a). Despite this, few MSI have the skills and means to effectively manage the transport, provide high quality service and keep the costs down (ANAP 2016a). With little expertise, MSIs are often led to adopt sub-optimal practices that impact costs, quality of service or the environment. Unlike medical transportation, medico-social transport is generally integrated into the life project of People With Disabilities (PWD), ANAP (2016b). Transportation is no longer considered merely a painful or non-value-added activity. It allows, the inclusion of the PWD to school and working life, on the one hand and, it is a lever for the autonomy, on the other hand ANAP (2016a).

Transportation demands in the medico-social sector are mostly regular and very often known in advance. This anticipated knowledge of needs can be used to jointly improve: the punctuality, quality of service for users, and the efficiency of the resources mobilized. The success of such a project requires the mobilization of all stakeholders in order to consider the different concerns of the parties involved. We must indeed meet the needs of people with disabilities and family carers, accompanying persons and mobility centers, MSI and professional carers.

Based on a field survey, conducted between 2017-2018 to 30 managers of MSI and 35 families from the Auvergne Rhone-Alpes Region in France, the purpose of this paper is to characterize the needs and objectives of the different stakeholders involved in the transport of PWD. This survey allows us to perform a processes mapping that presents the interactions of stakeholders in long, medium and short-term decisions. 
The following sections are organized as follows: In order to present the specificities and the existing methods of solving on-demand transport for PWD, Section 2 gives some bibliographical references. Section 3 presents the NOMAd project. Then, Section 4 describes the field study in three parts: the stakeholders, an analysis of needs drawn from a survey conducted in the fall of 2017 and a summary of the special features of the transportation for PWD. Section 5 illustrates where the decision support system is positioned within the general transportation process. Section 6 presents the general approach for constructing the annual transportation plan. Finally, the conclusions of this chapter are presented in Section 7.

\section{2 literature review}

The problem of on-demand transport for PWDs is already covered in the scientific literature as a single-period decision problem (Toth and Vigo 1997; Lehuédé et al. 2014). This problem belongs to the family of Diala-Ride transportation Problems (DARP) which is a generalization of the vehicle routing problem. However, our problem considers specific transport elements such as: the typology of transported people (wheelchairs or seated) (Parragh 2011) and the typology of adapted vehicles that can be modified in the depot only that is called configurable vehicle ( $\mathrm{Qu}$ and Bard 2015) or en-route modifications of the vehicle's inner configuration are allowed, that is called reconfigurable vehicle (Tellez et al. 2018).

As soon as we move from the single-period decision problem to a multi-period context, we must take into account the trajectory of decisions, including the overall service consistency. According to the literature, there are two types of service consistency: the driver-consistency, consisting in minimizing the number of different drivers for each user (Braekers, Caris, and Janssens 2014; Kovacs et al. 2015) and the time-consistency, consisting in reducing the dispersion of the timetable of each user. The time-consistency is considered in this paper.

The problem of time-consistency has already been treated in the scientific literature for a transportation problem close to ours but simpler. In Feillet et al. (2014), the problem is called "Time-Consistent Vehicle Routing Problem". It aims to optimize time-consistency in a context of a fleet of homogeneous vehicles, with the same destination for all passengers, and where no service time constraints are taken into account. The time-consistency for one user is defined as the number of different time slots where the user is picked up during the week. In our approach, a time slot can hold several service times within 10 minutes to each other. If one user has a different time slot each day, he has therefore 5 time-classes. A perfectly consistent schedule is characterized by a single time-class for each PWD. This optimal solution in terms of consistency is potentially too expensive for MSIs. So, it is important to find a balance between the quality of service which is measured with the time-consistency and the transportation costs. We call this problem the time-consistent DARP. To our knowledge, it has not yet been treated in the literature.

\section{The NOMAd project}

The project called "Numérique et Optimisation pour une Mobilité Adaptée" (NOMAd) is a 3-year project supported by the European Union through FEDER Founds. Deployed in the Auvergne Rhône-Alpes region, NOMAd aims to develop a web service to facilitate exchanges among all the stakeholders of adapted transport: transporters, families of PWD and MSIs. The aim is to build the transport solution by jointly integrating the points of view of the different actors (see https://nomad.disp-lab.fr/). Three objectives are targeted:

- Optimization of the transportation costs by reducing the number of kilometers traveled and the number of vehicles needed.

- Improvement of the quality of service for passengers and establishments by reducing travel times and provide time-consistent schedules.

- Reduce the environmental impact by reducing CO2 emissions linked to the number of kilometers traveled.

These objectives are interlinked and have been considered conjointly. As proposed in Zhu, Johnson, and Sarkis (2018), to be sustainable, the environmental impact reduction has to be associated with a cost improvement.

This project is carried out by a group of researchers from the DISP ${ }^{2}$ laboratory in collaboration with researchers from LS2N $\mathrm{N}^{3}$ laboratory and two strategic partners of the medico-social sector:

Ressourcial: a cooperative structure specialized in information systems for the medico-social sector. Created as an association, it is intended to share management practices and IT tools to non-profit MSIs.

\footnotetext{
${ }^{1}$ In English "Digital and Optimization for Adapted Mobility"

${ }^{2}$ https://disp-lab.fr/

${ }^{3}$ https://www.ls2n.fr/
} 


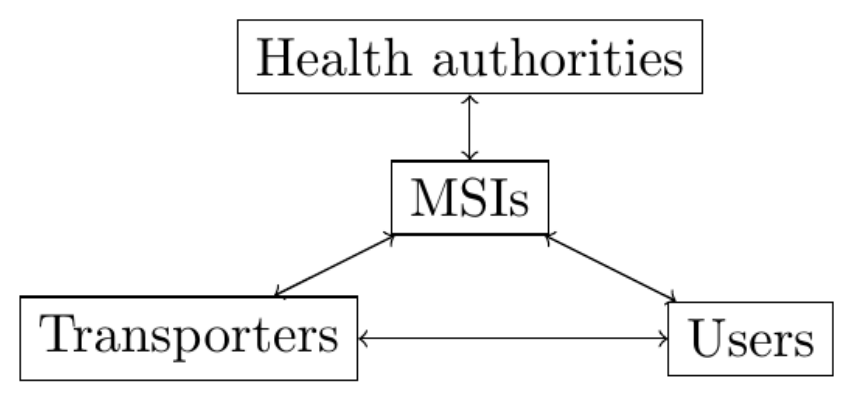

Figure 1: The four stakeholders in the transportation for PWD

SYNERGIHP Rhône-Alpes: a transportation company specialized in the management and realization of paratransit services. SYNERGIHP Rhône-Alpes intervenes particularly in the agglomeration of Lyon. To give an order of magnitude, this structure carries up to 1500 people per day. One of the benefits of SYNERGIHP Rhône-Alpes is that its stakeholders are the MSIs, which implies that making profit is not the only objective nor priority.

\section{Field study}

During the interaction with different stakeholders involved in the transportation for PWD we could understand the needs of the sector. In this section we present first the role of stakeholders, then an analysis of needs study drawn from a survey conducted in the fall of 2017 and finally a summary of the special features found in the transportation for PWD.

\subsection{Stakeholders}

In the process of transport for PWD there are multiple interdependent stakeholders assuring the reliability, quality and efficiency of the transportation service. As shown by Chakraborty (2018), a well-design interdependence can bring positive impact on the level of commitment between stakeholders.

Stakeholders can be classified into 4 categories: Health authorities, MSIs, Transporters and Users as shown in Figure 1. Arrows represent the direct interaction between stakeholders. Each category can include the coordination of multiple stakeholders.

\subsubsection{Health authorities}

Health authorities play the role of regulators and very often finance the transport itself. In France, most of MSIs are financed with public funds. This founds come from different sources, named: the National Solidarity Fund for Autonomy (CNSA) and health insurance (Assurance Maladie), and in a smaller proportions the State and Department councils.

\subsubsection{Medico Social Institutions (MSIs)}

MSIs accompany PWD daily in the construction of a life project that will allow PWD develop their autonomy. Some PWDs may stay in a boarding house the whole week, but the majority come back home daily. Each MSI receive a global budget to finance all its expenses, including transportation. Thus, there is an incentive to optimize costs without compromising the service quality. The transportation activity can be so cumbersome for MSIs that outsourcing is often seen as a promising solution. However, with an external transporter the coordination can be difficult and the quality of service may be compromised.

\subsubsection{Transporters}

The transportation activity can be internally organized by the MSI themselves or outsource to a specialized provider. In both cases, the transporter must follow strict regulations regarding safety measures, driving competences and vehicle specifications. The two main tasks for a transporter are: the construction of the annual transport planning and the daily operation of the transportation itself taking into account unforeseen events.

The transportation planning is a complex task that requires decision support tools. However, the available solutions in the market are scarce and do not completely adapt to the needs of the medico-social sector. 
Currently, complex tasks like route optimization are performed manually, as in SYNERGIHP Rhône-Alpes, based on field experience only. The project NOMAd is meant to fill these needs on in comprehensive manner.

\subsubsection{Users}

Users are PWD and their families who are very often the spokesperson. Families facilitate the transport of their relatives and stay in direct contact with MSI and the driver. In a certain way, families verify that the transportation is done with the expected quality.

\subsection{Survey: needs analysis}

In 2017, we conducted a survey with 30 managers of MSI (12 urban, 10 suburban and 8 rural) and 35 users (33 parents and 2 PSW) from the Auvergne Rhône-Alpes Region in France. This survey provides us an overview of the current situation of the adapted transportation from two different angles, from the users' perspective and from the service provider's perspective. The service providers are MSIs that can outsource the transportation activity. Nearly half of the MSIs surveyed outsource the transport.

\subsubsection{Service provider's perspective}

Figure 2 shows how MSIs perceive the quality of their transport and the resources they deploy in the transportation management. On one side, $77 \%$ of MSIs think they provide a good quality transport (yes + rather yes). This perception shows the importance given to the transportation activity. On the other side, this attention is confirmed with the resources deployed in the transportation management: $68 \%$ of MSIs put a great effort in the management of transport (large amount + a lot) as shown in the graph on the right.

Do you think you provide quality transport?

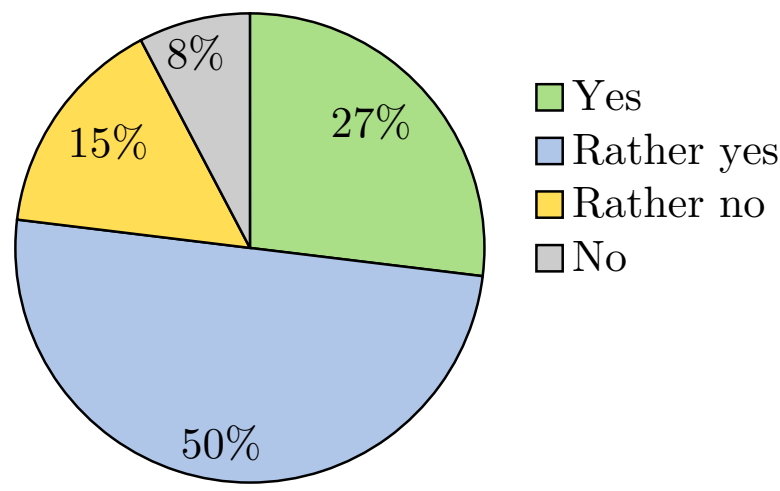

\section{How many ressources do you deploy in the transportation activity?}

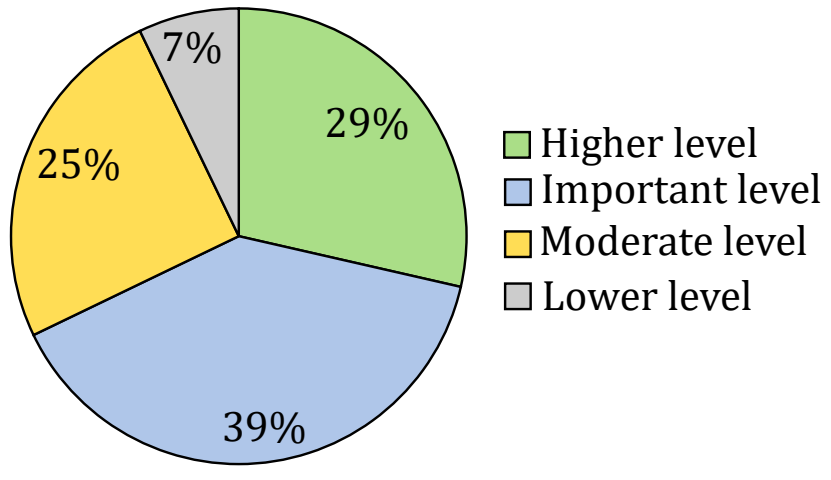

Figure 2: Transportation from the MSI perspective

For MSIs, the transportation activity is not just a logistics service, but it is integral part of the life project of PWD. The main resource required by the transportation activity is the staff necessary to the reception and departure; the realization of the transport itself, if it is not outsourced; and the staff for transportation management, gathering needs, planning routes and managing interactions with family caregivers on a daily basis. The last activities are often perceived as highly time-consuming and without real added value.

MSIs were also asked about the approximate distance of users to MSIs. Table 1 summarizes this distance in kilometers and minutes. Half of users live more than $30 \mathrm{~km}$ away from their MSIs and make more than 1 hour of ride time. Moreover, there is a high dispersion of demands reflected in a standard deviation of $46 \mathrm{~km}$ and 43 minutes. Note that these values are approximately estimated and sometimes overestimated by some administrators. This condition reinforces the interest to consider the transport pooling among several MSIs. This strategy is barely implemented in current practices. According to Rouquet and Vauché (2015), this type of pooling is known as District pooling, and leads to a more collective and stronger supply chain.

\subsubsection{Users' perspective}

Figure 3 shows the users view point regarding service quality on the left side and how users perceive their current ride time on the right side. Note however, that most of the responses come from parents as a spokesperson of PWDs. The perception of the service quality is very good 94\% ("yes" + "rather yes"). However, there is a $6 \%$ of users completely unsatisfied. This perception is a very clear opinion (no "rather no"). This situation is 
Table 1: Geographical dispersion of users

\begin{tabular}{lcc}
\hline & Distance $(\mathrm{km})$ & Time $(\mathrm{min})$ \\
\hline Median & 30 & 60 \\
Mean & 48 & 74 \\
Standard deviation & 46 & 43 \\
\hline
\end{tabular}

Are you satisfied with the service quality? Are you satisfied with the ride time?
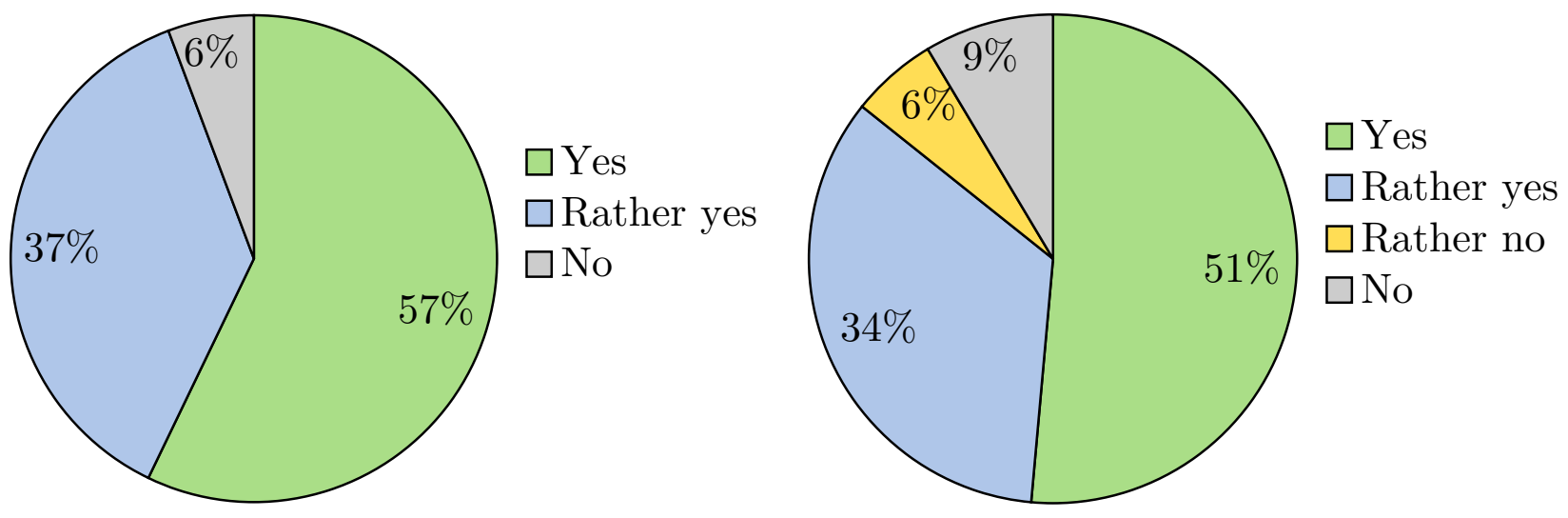

Figure 3: Transportation from the user's perspective

reflected in the graph of the ride side with $15 \%$ of users unsatisfied with their current ride time ("no" + "rather no"). This result shows us that one way to improve the quality of service is by reducing long ride times.

Users were asked to give a level of importance to different improvement directions. Figure 4 presents the results sorted from the most to the least important aspect. The most important aspect for users (parents) is to stay informed about modifications such as driver or scheduling changes (69\% very important). Users give more importance to the service punctuality than to have prior information about the vehicle arrival. Timeconsistency, which is having the same service time every day, seems to be a bit more important than driver consistency which is having the same driver every day.

\begin{tabular}{|l|c|c|c|c|}
\cline { 2 - 5 } \multicolumn{1}{c|}{} & Very important & Important & Moderate importance & Low importance \\
\hline Stay informed about unexpected events & $69 \%$ & $29 \%$ & $3 \%$ & $0 \%$ \\
Service punctuality & $43 \%$ & $49 \%$ & $9 \%$ & $0 \%$ \\
Time consistency & $34 \%$ & $40 \%$ & $17 \%$ & $9 \%$ \\
Driver consistency & $31 \%$ & $31 \%$ & $23 \%$ & $14 \%$ \\
Information about vehicle arrival & $14 \%$ & $29 \%$ & $46 \%$ & $11 \%$ \\
Make up suggestions & $11 \%$ & $71 \%$ & $11 \%$ & $6 \%$ \\
\hline
\end{tabular}

Figure 4: User's preferences

\subsubsection{Open questions}

In the survey to MSIs, we also address open questions regarding difficulties and possible actions to improve the transportation system. Regarding the main difficulties found in the transportation activity, managers of MSI point out the elements already seen above, ride times and transportation costs. They mention several other topics of concern:

- First, the need to take into account the specificity of this type of transport by adapting it to the PWD's needs. The necessity to manage behavioral problems in PWD like potential crises during transport.

- Secondly, the subject of communication and coordination especially for the management of unforeseen events, changes and delays. The coordination among 3 or more stakeholders and the need of real-time information about the transportation is a real concern for managers.

- Finally, managers evoke the human dimension of this transport. The need of a considerable number of 
staff in order to ensure the service quality and the safety. The specific driver training with respect to the qualifications and stability.

In regard of the actions to improve transportation, we found that $16 \%$ of the MSIs had integrated transportrelated objectives into their long-term contract with the health authorities (in France known as CPOM). MSI leaders mention the following objectives:

- To transform the transportation into a lever of autonomy for PWDs.

- To reduce operating costs, particularly those related to vehicle maintenance.

- To reduce ride times by the transport pooling among multiple MSIs.

- To adequately treat all the transport demands in the territory.

- To transform the transportation activity into an enabler of the PWD pesonal life project rather than a hurdle to his/her personal life project.

This field survey allowed us to define the three main objectives of the NOMAd project. It is about controlling costs, the quality of service and the ability to communicate and coordinate actions among multiple MSIs.

\subsection{A multidimensional problem}

The management and optimization of the transportation for PWD is a complex task. It requires taking into account a set of variables and features related to people, vehicles and the organizational scheme.

\subsubsection{Beneficiaries}

Beneficiaries are the PWD transported. They can be in a wheelchair or in a seat. PWD have a certain degree of autonomy but some have to be accompanied by a third party. From a quality of service perspective, the time dimension is very important. Users have availability constraints that must be respected. These constraints are expressed in the form of time windows with the earliest and latest arrival time. A maximum ride time of transport for each person is taken into account. Furthermore, in the design of routes a consistent schedule must be taken into consideration. Indeed, despite the variability of demand during the week, it is better to give a single time of service in the morning (afternoon) when they are present several days during the week.

\subsubsection{Vehicles}

Vehicles used in the transport for PWD are specifically designed. Each vehicle can receive a limited capacity for people in wheelchair and/or seated. Additionally, one vehicle may have different layouts that can be configured before the departure or during the routing. These configurations are made with retractable seats, for example, to swap a wheelchair space with a conventional seat. Figure 5 shows four different layouts of a reconfigurable vehicle $^{14}$

This vehicle can have spaces for seats, wheelchairs and electric wheelchairs(in black).

\subsubsection{Co-organization}

Stakeholders are characterized for having different, even antagonistic, objectives. A transport management based solely on a financial criterion will tend to reduce the number of vehicles mobilized and to offer rather long routes, which is a sub-optimal policy from a quality point of view. Conversely, an organization of a transport seeking only to offer the best quality of service will tend to increase the number of vehicles and to offer directroute transport for each person. Thus, it seems necessary to find a compromise solution. A multi-criteria approach is therefore required to take into account together cost reduction, environmental impact and quality of service criteria.

\section{Transportation management processes}

There are multiple processes involved in the management of transport for PWD. Figure 6 shows the Business Process Modeling (BPM) of the main transportation activities. In it, we model the interaction among the MSI, the transporter and users in order to perform 3 main processes.

(i) The transport mutualization to determine the opportunities of transport pooling between MSIs;

\footnotetext{
${ }^{41}$ source: https://www.handynamic.fr/
} 


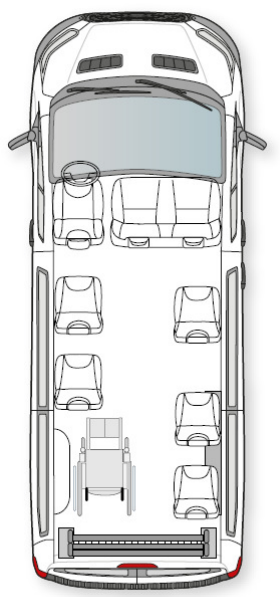

8 闹+1国

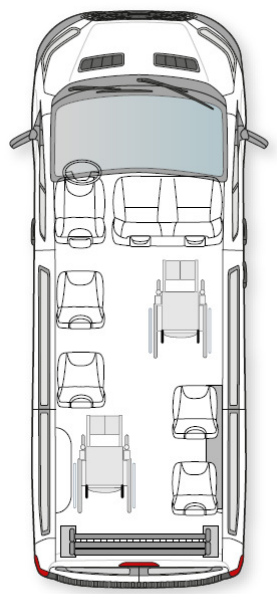

7 闹十

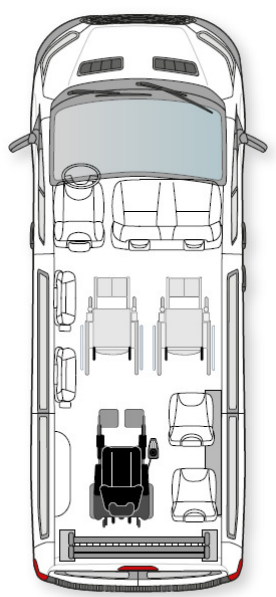

5 闹+3

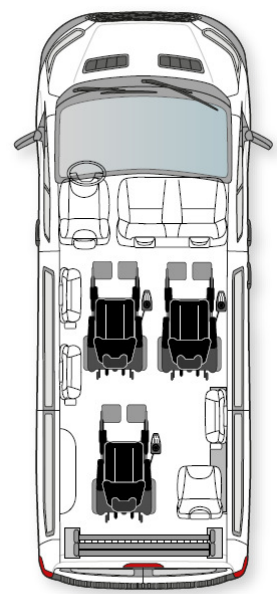

4 闹+3

Figure 5: Example of a reconfigurable vehicle

(ii) the long-term planning processes defining the annual transportation plan of each user and logistics need like the fleet;

(iii) the short-term planning processes to manage the day-to-day modifications of the transportation plan; and,

(iv) the transport execution process covering the daily iterations between stakeholders and the follow up of routes.

The scientific results of the following section respond to the need of decision support tool for the long-term planning. It is bounded with a dotted square in the Figure 6. 


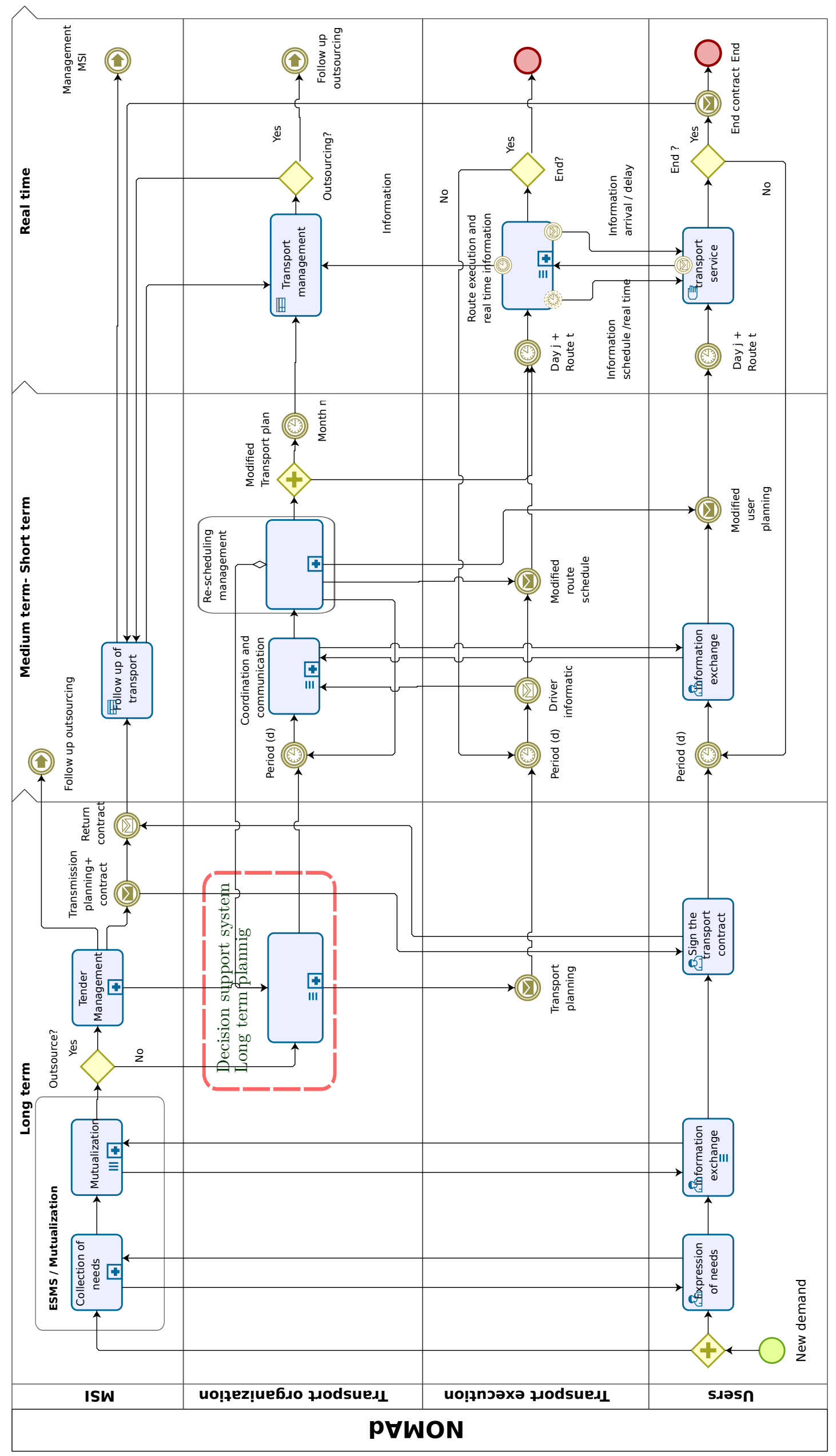

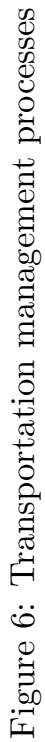




\section{Construction of the long-term transportation planning}

The construction of the long-term transportation planning is a complex problem that has not been completely solved in scientific literature. The long-term planning considers one academic year. In accordance with practice, we assume that this transport plan is constructed from a pattern of identical weekly transportation plans.

This section presents the process of building a typical week. Defining a weekly schedule is a complex task that needs to be decomposed into sub-problems. First we work in the planning of each sub-problem called the half-day transportation problem. Secondly, we focused on building the weekly schedule from the sub-problems that compose it. Figure 7 illustrates the process. The rectangle $M_{1}$ corresponds to one sub-problem related to the half-day problem. The dotted rectangle specifies the perimeter of the weekly morning or afternoon schedule. We therefore assume that the problem of the morning and the afternoon can be solved independently.

\begin{tabular}{|c|c|c|c|c|c|}
\hline & Monday & Thuesday & Wednesday & Thursday & Friday \\
\hline Morning & $\mathrm{M}_{1}$ & $\mathrm{M}_{2}$ & $\mathrm{M}_{3}$ & $\mathrm{M}_{4}$ & $\mathrm{M}_{5}$ \\
\hline Afternoon & $\mathrm{A}_{1}$ & $\mathrm{~A}_{2}$ & $\mathrm{~A}_{3}$ & $\mathrm{~A}_{4}$ & $\mathrm{~A}_{5}$ \\
\hline
\end{tabular}

Figure 7: Structuring of a typical week

\subsection{Building the half-day transportation schedule}

Defining the half-day schedule consists in solving an extension of the so-called Dial-a-Ride transportation Problem (DARP). In the DARP, each person transported is characterized by having a different pickup and delivery location, two time windows and a maximum ride time. Vehicle capacity constraints are also integrated. The transport optimization of the half-day problem is based on a cost objective that incorporates vehicle depreciation, hourly costs (e.g. driver wages), and mileage costs. The respect of pickup and delivery time windows and maximum ride time is expressed as timing constraints. Figure 8 illustrates two morning routes for transporting PWD to MSI. Pickups are illustrated by blue houses and deliveries on MSIs by gray ones. Pickup can concern only one PWD (on wheelchair or not) or several people. In the last case, this pickup represents an aggregation point. Thanks to transport pooling, routes are able to visit several MSIs.

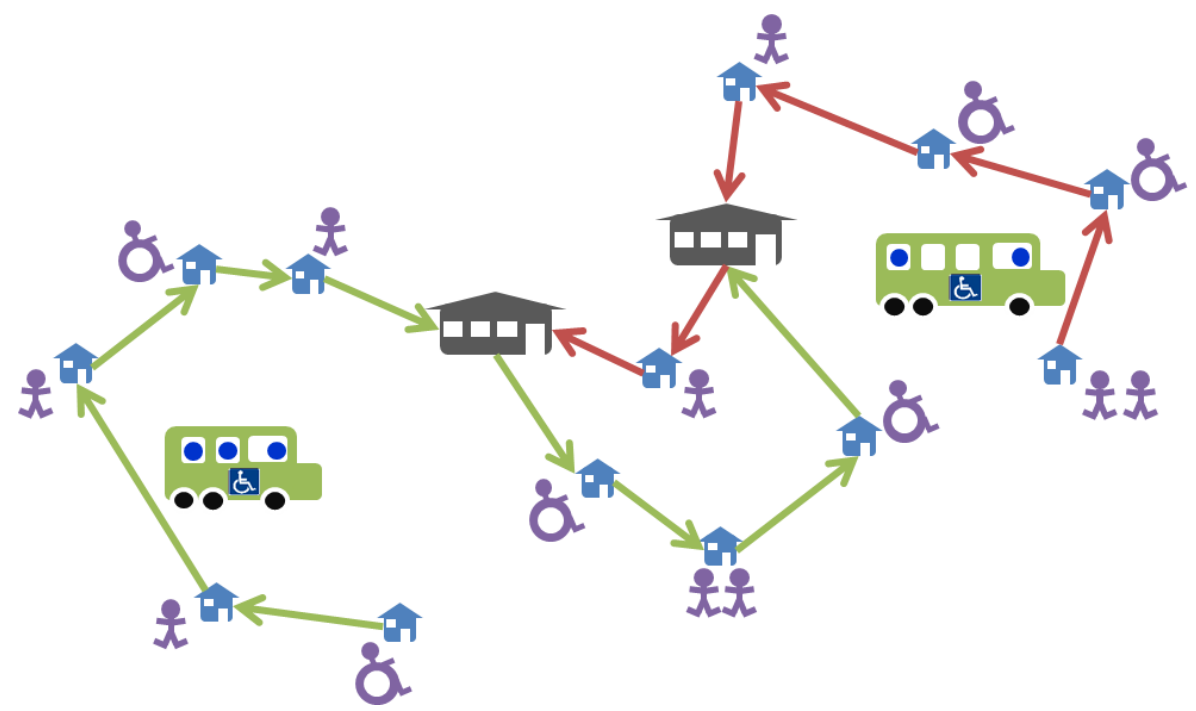

Figure 8: Illustration of vehicle routes

The DARP using vehicles similar to the one presented in Section 4.3.2 was studied in the scientific literature. This problem is known as the fleet size and mix dial a ride problem with reconfigurable vehicle capacity. The size and type of fleet is determined and at the same time as the routes. In our work, this problem is solved using the matheuristic LNS-RSCP proposed in Tellez et al. (2018). This matheuristic combines a Large Neighbourhood Search procedure with a Set Covering component to find high quality solutions at a reasonable time. 


\begin{tabular}{ll}
\hline$y_{\omega}^{t} \in\{0,1\}$ & $=1$ if route $\omega \in \Omega$ is selected at period $t \in \mathcal{T}$, and 0 otherwise \\
$\mu_{u c} \in\{0,1\}$ & $=1$ if user $u \in \mathcal{U}$ uses time-class $c \in \mathcal{C}$, and 0 otherwise \\
$v$ & number of vehicles needed for the whole planning horizon \\
$m_{u}$ & number of users having $c \in \mathcal{C}$ time-classes (post-processed variable) \\
\hline
\end{tabular}

Table 2: Variables

\subsection{Time-consistency on the week schedule}

Once we solved the half-day transport problem with the matheuristic LNS-RSCP, we conduct a weekly solving. Indeed, not everyone needs to be transported every day. The simple juxtaposition of half-day transportation schedules can give a very efficient transport planning. However, in this plan, users may have a different service time every day. This situation is uncomfortable for PWD, or even unacceptable for some people with mental disabilities. Thus, from a global approach this weekly schedule aims to both control costs and time-consistency throughout the week.

The week schedule problem can be modeled as a classical DARP for each period of the week (Tellez et al. 2018). The specificity lies in the multi-objective formulation.

\subsubsection{Objectives of the week schedule}

We consider $\mathcal{U}$, a set of PWD, to be transported during a planning horizon $\mathcal{T}$. Each PWD $u \in \mathcal{U}$ has a demand indicator $\beta_{u}^{t} \in\{0,1\}$ for each period $t \in \mathcal{T}$. We also consider a set of routes $\omega \in \Omega$, that are potentially used to satisfy transport demand of PWD during the planning horizon $\mathcal{T}$. Each route $\omega \in \Omega$ serves a subset of PWDs. Each route $\omega \in \Omega$ is operated by one vehicle and has a variable cost $C_{\omega}$. To each vehicle is also associated a weekly ownership cost $\lambda$. The total vehicle ownership cost depends on the number of vehicles $v$ needed during the whole planning horizon.

The decision problem can be seen as a bi-objective problem which consists in selecting a subset of routes from $\Omega$ such that transportation requests on the planning horizon are satisfied within their time windows and maximum ride times. The first objective is to minimize the transportation costs. The second objective is to minimize the service time inconsistency.

To model the problem, we introduce the binary decision variable $y_{\omega}^{t}$ which is equal to 1 if route $\omega \in \Omega$ is selected at period $t \in \mathcal{T}$. We model time-classes as proposed in Feillet et al. (2014) by defining a set $\mathcal{C}$ of different time slots during which the PWD is picked up during the planning horizon $\mathcal{T}$. We also used binary variables $\mu_{u c}$ to indicate which time-classes from set $\mathcal{C}$ are actually used by user $u$. In a 5 days week, the number of time classes used is at most 5. If $u$ has 3 time-classes, we assume that time-classes 1,2 and 3 are used, and time-classes 4 and 5 are not used. Hence, $\mu_{u, 1}=\mu_{u, 2}=\mu_{u, 3}=1$.

Table 2 shows variables used to define the objectives of mathematical model.

The first objective is the minimization of transportation costs $f$ that are defined by the sum of fixed and routing costs. Fixed costs are related to the cost of owning the vehicles. Then, the cost $C_{\omega}$ of a route $\omega \in \Omega$ depends on its duration and on its length.

$$
\min f=\lambda v+\sum_{\omega \in \Omega} \sum_{t \in \mathcal{T}} C_{\omega} y_{\omega}^{t}
$$

The second objective minimizes time inconsistency $\boldsymbol{g}$, which is modeled with a lexicographical refinement of the time-class model proposed by Feillet et al. (2014). The expression used in the MILP model is the following:

$$
\operatorname{lexmin} \boldsymbol{g}=\left(\sum_{u \in \mathcal{U}} \mu_{u|\mathcal{C}|}, \ldots, \sum_{u \in \mathcal{U}} \mu_{u 2}\right) .
$$

This expression lexicographically minimizes the number of people having more than $c$ time-classes, where $c$ decreases from $|\mathcal{C}|$ to 2 . The expression $\sum_{u \in \mathcal{U}} \mu_{u c}$ counts the number of users with $c$ or more time-classes. This is equivalent to the lexicographical minimization of the number of users whose number of time-classes is exactly $|\mathcal{C}|,|\mathcal{C}|-1$, down to 1 , respectively. The MILP constraints are the classical constraints of the DARP. For the sake of conciseness, we do not detail them here.

\subsubsection{Solution method}

Figure 9 illustrates the proposed solving approach for the time-consistent DARP. We begin by solving the subproblem of each half-day described in Section 6.1. The union of these solutions is the starting point for the construction of the weekly transportation plan. This first plan is economically efficient, but highly inconsistent. 
During this first step, we collect the routes obtained in the different iterations to constitute a pool of routes useful to the improvement of time-consistency. Then, using an optimization MILP solver, a time-consistent DARP is solved using a subset of routes from the pool. A solution provides the service times of each day and the time-classes of each user as in shown in Solution $S$ of Figure 9. The small vertical lines represent the service times of the week and the gray area the time-classes.

This process is repeated iteratively using each time a different subset of routes until a local optimum is reached. Then a diversification procedure (red arrow) is triggered to generate new routes compatible with the time-classes of the current solution. The diversification procedure defines first custom multiple time windows for each user, and then solves a DARP for each day. The routes generated through the solving enrich the pool of routes.

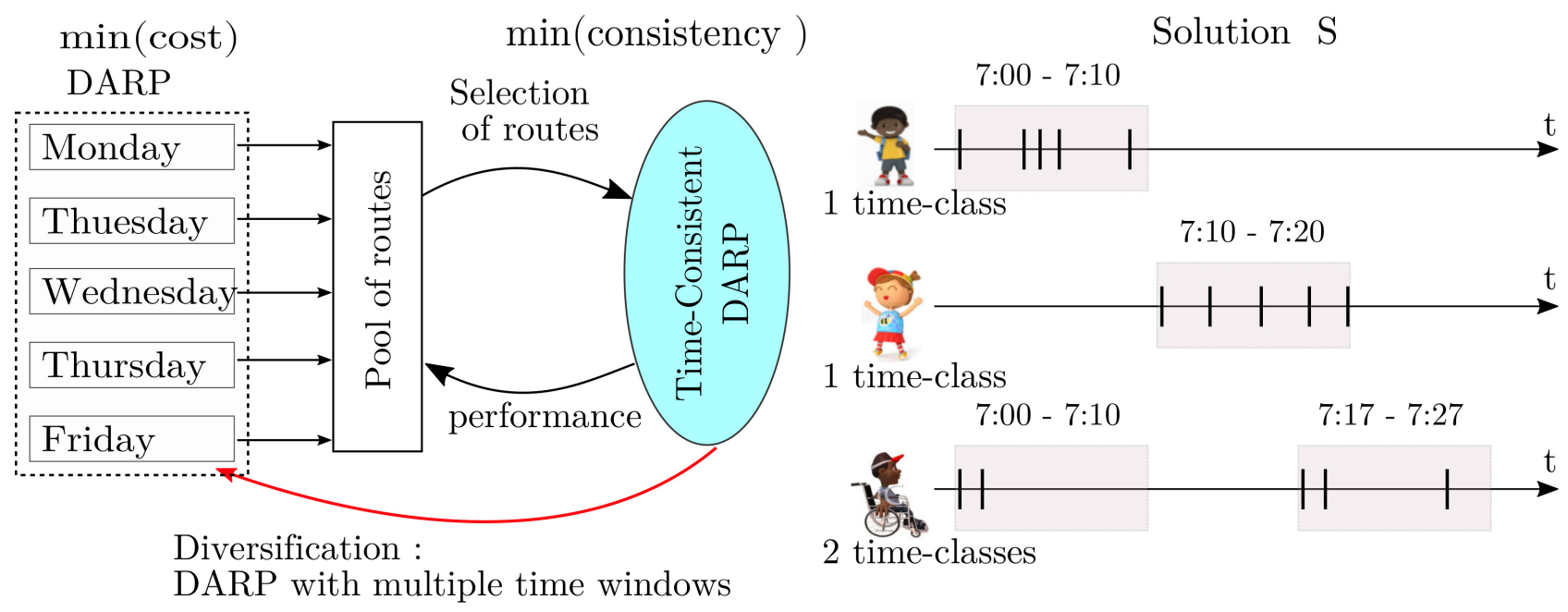

Figure 9: Global approach to improve time-consistency

In order to find the link cost and time-consistency, we construct a Pareto front using an epsilon-constraint approach. Each new solution of the front is found by relaxing the cost of the previous solution by an epsilon quantity in order to gradually improve the time-consistency.

Figure 10 shows the Pareto front for an instance of 135 users. We collected real data from the SYNERGIHP Rhône-Alpes. This data concerns the transportation of users (PWD) to 16 MSIs. Each point corresponds to a different solution. The $\mathrm{y}$-axis presents the time inconsistency and $\mathrm{x}$-axis the cost increase (\%) with respect to the cheapest solution found. The time inconsistency is shown in vectorial form. The cheapest solution has an inconsistency of $[6,38,91]$ that is 6 users with 3 time-classes, 38 with 2 time-classes and 91 users with 1 timeclass (First point on the left). In this example we can see that a slight cost increase of $0.5 \%$ can significantly improve the solution quality as none of passengers have more than 2 time-classes $[0,30,105]$. Finally, note that reaching a perfect consistency $[0,0,135]$ increase the cost up to $8.5 \%$.

\section{Conclusion}

In the NOMAd project, we investigated the problem of transport of people with disabilities. Its management and its optimization are complex tasks. This paper is focused on a study of the several stakeholders' needs and constraints. This analysis shows two important objectives: reducing transportation costs and improving quality of service. In order to understand the transportation management processes, we propose a BPM model in which the four stakeholders' interactions are formalized. For medico-social institutions, it is a highly demanding task and very often requires a lot of human resources to ensure quality and safety. Knowing that this transport is characterized by a strong geographical dispersion of users, there is a real economic interest in optimizing and pooling this service. This must be done without degrading the quality of service.

The main decision problem is the long-term planning. It requires taking into account a set of variables and specificities related to people, vehicles and organizational schemes. To this end, we propose a comprehensive approach for developing a long-term transport planning that is both cost-effective and time-consistent for people with disabilities. This problem is solved in two steps. First, the half-day transportation schedule is built with a cost optimization objective. Second, the ten half-days are compiled to propose the outline of the week schedule optimizing the time-consistency and transportation costs. The first step is a specific dial-a-ride problem that is solved by using a matheuristic based on Large Neighbourhood Search and a Reactive Set Covering Problem. The second step is a bi-objective time-consistent dial a ride problem which is solved by using an epsilon-constraint 


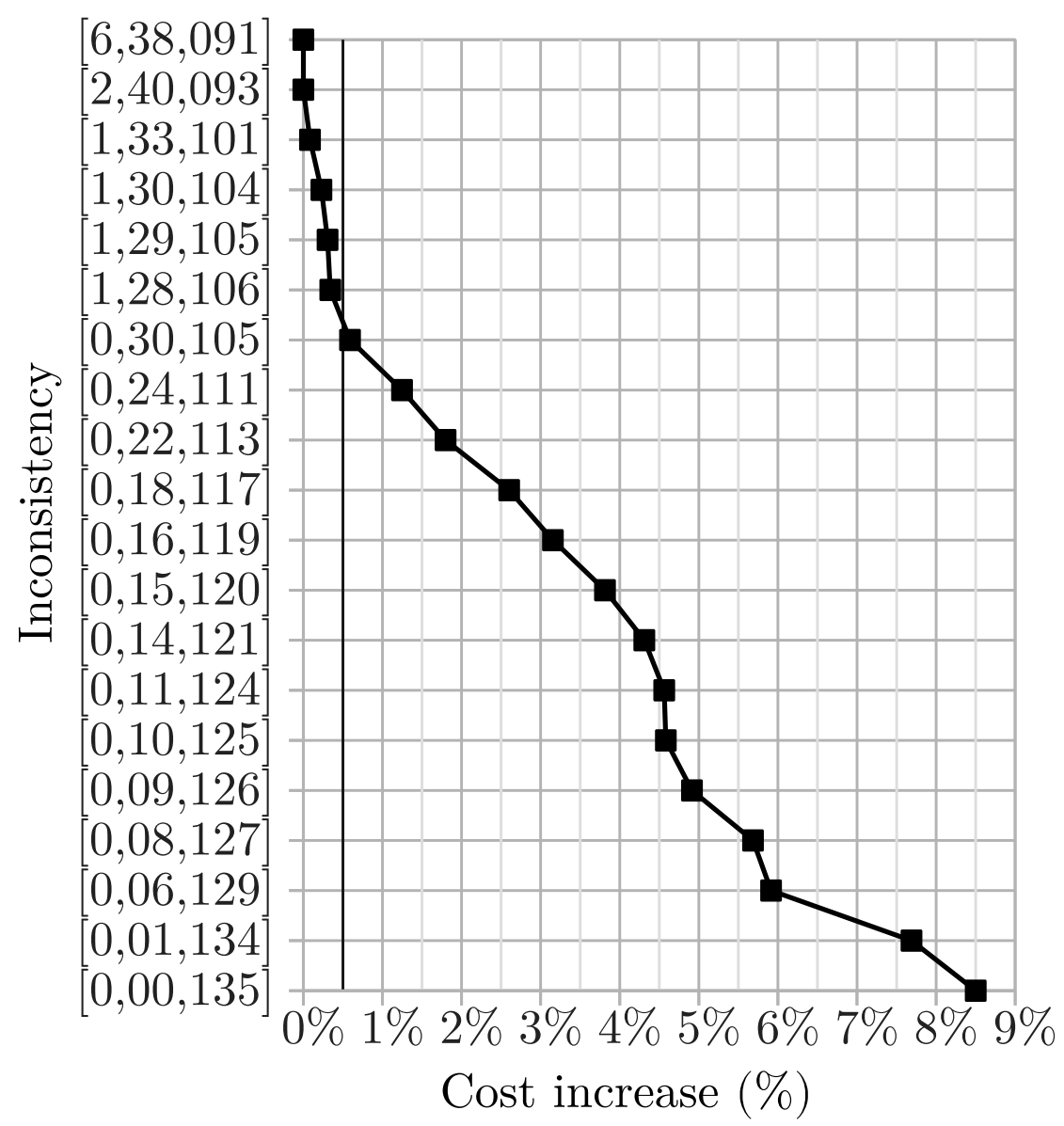

Figure 10: Pareto front solution with 135 passengers

approach.

Future research includes the extension of the work to short-term decisions. Then, incorporating real-time information (traffic, variable demand) may require the use of state-space-time networks with a fine time discretization (see, e.g. Mahmoudi and Zhou (2016)) and specific optimization algorithms such as the Dynamic Discretization Discovery (Boland et al. 2017).

\section{Funding}

We would like to thank the European Union through the European regional development fund (ERDF) and the French region Auvergne-Rhône-Alpes for their financial support of the NOMAd project.

\section{References}

ANAP. 2013. Améliorer la gestion des transports sanitaires en établissement de santé. Technical Report. Appui santé \& médico social.

ANAP. 2016a. Améliorer la gestion des transports de personnes handicapées. Tome 1: Rétour d'expériences. Technical Report. Appui santé \& médico social.

ANAP. 2016b. Améliorer la gestion des transports de personnes handicapées. Tome 2: Modalités organisationnelles. Technical Report. Appui santé \& médico social.

Boland, Natashia, Mike Hewitt, Luke Marshall, and Martin Savelsbergh. 2017. "The Continuous-Time Service Network Design Problem." Operations Research 65 (5): 1303-1321.

Braekers, Kris, An Caris, and Gerrit K. Janssens. 2014. "Exact and meta-heuristic approach for a general heterogeneous dial-a-ride problem with multiple depots." Transportation Research Part B: Methodological 67: 166-186. 
Chakraborty, Samyadip. 2018. "Enablers of co-creation in hospital-supplier relationships: empirical study in Indian healthcare context." In Supply Chain Forum: An International Journal , Vol. 19 (4): 331-352. Taylor \& Francis.

Feillet, Dominique, Thierry Garaix, Fabien Lehuédé, Olivier Péton, and Dominique Quadri. 2014. "A new consistent vehicle routing problem for the transportation of people with disabilities." Networks 63 (3): $211-224$.

Gonzalez, Lucie, Romain Roussel, Jean-Cyprien Héam, Myriam Mikou, and Carine Ferretti. "Les dépenses de santé en 2017 - Résultats des comptes de la santé - Édition 2018" In Panoramas de DREES Santé.

IGF/IGAS. 2012. Établissements et services pour personnes handicapées offre et besoins, modalités de financement. Technical Report. Inspection Générale des Finances \& Inspection Générale des Affaires Sociales.

Kovacs, Attila A., Bruce L. Golden, Richard F. Hartl, and Sophie N. Parragh. 2015. "The Generalized Consistent Vehicle Routing Problem." Transportation Science 49 (4): 796-816.

Lehuédé, Fabien, Renaud Masson, Sophie N Parragh, Olivier Péton, and Fabien Tricoire. 2014. "A multi-criteria large neighbourhood search for the transportation of disabled people." Journal of the Operational Research Society 65 (7): 983-1000.

Lesteven, Robert, Wahl, and Grimonprez. "Les transports sanitaires - Revue de dépenses 2016" In Rapport $I G A S$.

Mahmoudi, Monirehalsadat, and Xuesong Zhou. 2016. "Finding optimal solutions for vehicle routing problem with pickup and delivery services with time windows: A dynamic programming approach based on state-space-time network representations." Transportation Research Part B: Methodological 89: 19-42.

Parragh, Sophie N. 2011. "Introducing heterogeneous users and vehicles into models and algorithms for the dial-a-ride problem." Transportation Research Part C: Emerging Technologies 19 (5): 912-930.

Qu, Yuan, and Jonathan F. Bard. 2015. "A Branch-and-Price-and-Cut Algorithm for Heterogeneous Pickup and Delivery Problems with Configurable Vehicle Capacity." Transportation Science 49 (2): 254-270.

Rouquet, Aurélien, and Lucie Vauché. 2015. "A typology of logistics pooling in supply chains." In Supply Chain Forum: An International Journal, Vol. 16, 2-12. Taylor \& Francis.

Tellez, Oscar, Samuel Vercraene, Fabien Lehuédé, Olivier Péton, and Thibaud Monteiro. 2018. "The fleet size and mix dial-a-ride problem with reconfigurable vehicle capacity." Transportation Research Part C: Emerging Technologies 91: $99-123$.

Toth, Paolo, and Daniele Vigo. 1997. "Heuristic algorithms for the handicapped persons transportation problem." Transportation Science 31 (1): 60-71.

Zhu, Qingyun, Sharon Johnson, and Joseph Sarkis. 2018. "Lean six sigma and environmental sustainability: a hospital perspective." In Supply Chain Forum: An International Journal, Vol. 19, 25-41. Taylor \& Francis. 